Review Article

\title{
Chronic fatigue syndrome/post traumatic stress disorder: are they related?
}

Volume 7 Issue 2 - 2017

Keywords: chronic fatigue syndrome, disease control, cdc, symptoms, neuroendocrine system, post traumatic stress disorder

Abbreviations: CDC, centers for disease control; MPS, myofascial pain syndrome; CFS, chronic fatigue syndrome; PCI, pain coping inventory; FIQ, fibromyalgia impact questionnaire; QEEG, quantitative EEG; EEG, electroencephalograph

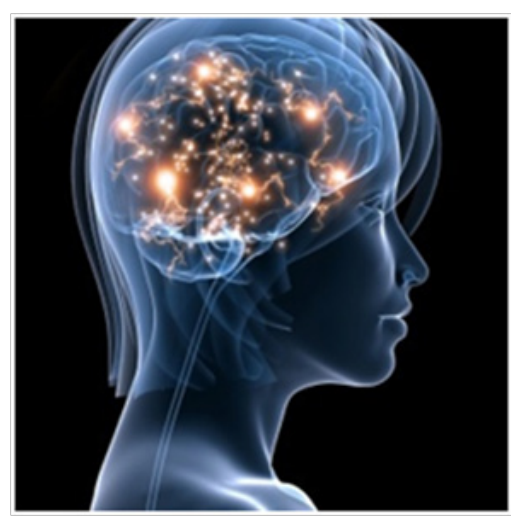

\section{Introduction}

Chronic Fatigue Syndrome/Fibromyalgia remains an enigma, with current research focusing on numerous and varying aspects of the conditions. As noted by the Centers for Disease Control (CDC) in the US National Library of Medicine's Medline Plus: "No one knows what causes CFS. It is most common in women in their $40 \mathrm{~s}$ and $50 \mathrm{~s}$, but anyone can have it. It can last for years. There is no cure for CFS, so the goal of treatment is to improve symptoms".

A June 2015 article in the Annals of Internal Medicine represents a seismic shift in how some in the medical community have altered their perception of the CFS condition. ${ }^{2}$

According to the most widely used case definition, the illness is characterized exclusively by symptoms; therefore, physicians have understandably wondered whether there are "real" underlying biological abnormalities. The IOM, AHRQ, and NIH panels concluded that there are such biological abnormalities. After evaluating thousands of published articles, the IOM committee stated that "ME/ CFS is a serious, chronic, complex systemic disease that often can profoundly affect the lives of patients.

Summarizing the committee's deliberations, Ganiats in redefining the chronic fatigue syndrome said that the illness: "is not, as many clinicians believe, a psychological problem," while emphasizing that psychiatric comorbid conditions occur in some patients with ME/ CFS and need to be diagnosed and treated (Ann Intern Med. 2015; 162:653-4).

As noted by Charles W. Lapp, MD, "Other 'shadow syndromes' include premenstrual syndrome, primary dysmenorrhea, migraine,

\author{
George Lindenfeld,' George Rozelle, ${ }^{2}$ \\ Katherine Billiot ${ }^{3}$ \\ 'Diplomate in Clinical Psychology, Medical Psychology Center in \\ Asheville, USA \\ 2Diplomate in QEEG and Neurotherapy, USA \\ ${ }^{3} \mathrm{BCIA}$ Certified in Biofeedback \& Neurofeedback, USA
}

Correspondence: George Lindenfeld, Diplomate in Clinical Psychology, Medical Psychology Center in Asheville, USA, Email glindy123@gmail.com

Received: July 22, 2016 | Published: February 08, 2017

restless legs or periodic leg movements, Myofascial Pain Syndrome (MPS), chemical sensitivities, autonomic dysfunction (Neurally Mediated Hypotension and Postural Orthostatic Tachycardia Syndrome), Gulf War Syndrome, and possibly hyper extensibility, acne rosacea, Raynaud's phenomenon, sicca complex (dry eyes and mouth) and vasomotor rhinitis (constant runny nose and congestion)". ${ }^{3}$

Rather than addressing each of the above conditions, we have chosen to target our focus specifically to Chronic Fatigue Syndrome (CFS). Our intent is to use CFS as a primary model with the underlying belief that other conditions, such as those discussed above, share multiple common features. We take the position that the terms Chronic Fatigue Syndrome and Fibromyalgia can be used interchangeably to address the same condition at this point in time based on review of recent articles and studies. We provide a number of such articles to support our perspective.

There is an increasing interest in understanding the biological mechanism underpinning fibromyalgia (FM) and chronic fatigue syndrome (CFS).

Despite the presence of mixed findings in this area, a few biological systems have been consistently involved, and the increasing number of studies in the field is encouraging. This chapter will focus on inflammatory and oxidative stress pathways and on the neuroendocrine system, which have been more commonly examined. Chronic inflammation, together with raised levels of oxidative stress and mitochondrial dysfunction, has been increasingly associated with the manifestation of symptoms such as pain, fatigue, impaired memory, and depression, which largely characterise at least some patients suffering from CFS and FM. 
Furthermore, the presence of blunted hypothalamic-pituitaryadrenal axis activity, with reduced cortisol secretion both at baseline and in response to stimulation tests, suggests a role for the hypothalamic-pituitary-adrenal axis and cortisol in the pathogenesis of these syndromes. However, to what extent these systems' abnormalities could be considered as primary or secondary factors causing FM and CFS has yet to be clarified. ${ }^{4}$

The authors of the next 2015 review focused on current literature related to central sensitization and physiological studies in the functional somatic syndromes.

The results of the inquiry suggested that central sensitization may be a common neurophysiological process that is able to explain nonpainful as well as painful symptoms in these disorders. Furthermore, central sensitization may represent an endophenotypic vulnerability to the development of these syndromes that potentially explains why they cluster together.

The organizational flow of this current case study begins with our perspective that shared features of both CFS as well as PTSD primarily include the collapse of systemic coping mechanisms that deal with prolonged stress. We further propose that a neuronal system reaction takes place that transforms the afflicted individual into a chronic defensive/reactive mode rather than a normative state of growth.

Specifically, we question whether CFS is a corresponding condition to PTSD wherein the brain remains in a constant state of vigilance that is incurred through a different, subtle form of traumatic or physiologically induced distress? Might underlying emotional disturbance or its equivalent produce the brain inflammation referred to in Chronic Fatigue Syndrome ${ }^{6,7}$ ? Alternatively, might the condition be due to something utterly different from trauma such as infection, tick bite, etc.

L. Richard Bruursema and I posted a paper on Academia.edu entitled "Resetting the Fear Switch in PTSD: A Novel Treatment Using Acoustical Neuromodulation to Modify Memory Reconsolidation". ${ }^{8}$ Furthermore, a second paper by Dr. George Lindenfeld and George Rozelle followed in Academia.edu pertaining to a case study that described a rapid remediation of PTSD symptomology. The article is entitled "PTSD: Brain on Fire: A RESET Therapy QEEG Brain Map Analysis of an Afghanistan Combat Veteran". ${ }^{\text {" }}$

The present paper is based upon the application of the principles elucidated in the Resetting the Fear Switch and Brain on Fire papers. Specifically, we postulated that RESET Therapy (Reconsolidation Enhancement by Stimulation of Emotional Triggers) interrupts reconsolidation of emotionally charged material in the limbic system through a neuro-modulation process, thereby resulting in rapid and dramatic relief of PTSD symptoms. We ask, in regards to CFS, might the same intervention be effective in a condition that may induce a state of chronic stress that emanates from a possible non-trauma source?

Our earlier hypothesis was that when hyper-aroused/hypersensitized neuronal circuits are interrupted through an acousticallydriven neuromodulation process, they appear to 'reset' back to (or closer to) a norm that existed prior to the trauma experience. Both the normal and disturbed states may be homeostatic, i.e., modulated around a set point. In the disturbed state, the set point may be quite different from the norm and/or the modulation may be much greater or much smaller. The neural reset is evidenced by the lasting reduction or elimination of the reported symptoms. We believe that RESET
Therapy enables the brain to re-establish plasticity that became frozen through the effects of trauma or chronic stress factors. This testable hypothesis will be applied to our CFS focus within the context of a case study that follows this discussion.

We believe that two critical components of RESET Therapy are common to all emerging memory, chronic stress and trauma based therapies. The first objective is to re-stimulate the reactive limbic portion of the selected neural circuit through the patient's sustaining of attentional and sensory focus. The second aspect is for the therapist to introduce a modifying stimulus: in our case a uniquely tuned in pulsed sound that interrupts the reconsolidation process of the hyperactivated or sensitized memory circuit.

The instrument developed by Dr. Frank Lawlis utilized to accomplish the above objectives is called the BAUD (http:// franklawlis.com, http://baudenergetics.com). Itproduces a full square wave which is typically perceived by the patient as unpleasant or even somewhat irritating. It has been compared to the sound of angry, buzzing bees. We believe that this reaction elicits an amygdala-based emotional arousal. The cortical pathway of the mechanism of action for this treatment is thought to be as follows: research has shown that sound has a direct, unfiltered pathway to the amygdala through the auditory thalamus (medial geniculate nucleus). It is well-established that sound, especially aversive sound, has an arousing effect on the amygdala. ${ }^{10}$ A common example of this would be the startle response, where a loud sound makes us duck without thinking.

We perceive that through the acoustical pathway we are able to directly modulate neural activity in the amygdalo-hippocampal circuit during memory retrieval and reconsolida-tion. Emotional memories are first consolidated here marked by synchronized theta activity between the amygdala and hippocampus and are reactivated during fear memory retrieval. ${ }^{11}$ The combination of the patient's multifaceted attention with the BAUD's acoustical theta stimulation during the reactivation process creates a state of synaptic plasticity which provides a unique opportunity for therapeutic intervention.

We perceive that when resonance is established between the patient's selected target frequency and the properly adjusted pulsed sound emanating from the BAUD, normalization may take place immediately or within hours following the first actual treatment session. A supposition based on the Neuronal Model of PTSD, as described in Resetting the Fear Switch, is that when resonance occurs through an externally induced neuro-modulated sound, reactivation of cortical circuitry will take place in the prefrontal region of the brain as well as in the speech centers (Brocha's and Werniche's) in the left hemisphere. ${ }^{12}$

Additionally, we proposed that the limbic system will return to a normal homeostatic balance as evidenced in our PTSD treatment findings. Translated, this implies that the patient with CFS will experience a calming effect manifested in a release of tension that has locked in throughout the brain's involved neuronal network and the body's muscle fiber tract system. We have also come to expect that the patient will become much better in fully articulating her emotionally charged material after her brain has been RESET rather than before. Clinically, this has been the case each and every time for those PTSD patients who have experienced this treatment.

We now speculate that with our CFS case study patient (Lynn), her amygdala and other components of her limbic system were locked in an activation mode that placed her into a likely state of chronic freeze. Based on our neuronal model, we anticipated that her limbic system 
disturbance would deactivate following RESET Therapy, thereby gradually returning her to pre-onset cognitive and physiological levels. We reference this phenomenon as 'turning off the neuronal switch'thereby permitting the individual to experience a sense of inner calmness free of physiological reactivity.

Prior to providing you with our case study, we summarize briefly here our earlier and recent clinical experience with CFS patients. Following a traditional biofeedback/ neuro feedback practice, we found ourselves becoming increasingly frustrated with the "no show' frequency of those afflicted with this disorder because of their frequent state of immobility. Ultimately, as solo practitioners, we decided that we were no longer able to accept the referral of patients with this condition because of this seeming non-compliance factor.

With the use of RESET Therapy, our experience and perception of this condition have altered dramatically. We have found that RESET Therapy is ideally suited to treat CFS in the home setting rather than requiring the patient to attend scheduled outpatient treatment appointments. Where possible, we provided 'house calls' (call us old fashioned) to educate the patient and demonstrate the proper use of the BAUD to 'tune in' to the selected target. Consequently, with the patient obtaining a BAUD for home use, treatment can occur anytime thereafter based on the patient's status and capacity rather than by the therapist's rigid scheduling requirements. Interchange can take place through telephone, Skype or e-mail contact.

We expect the flow and progress with CFS to be more akin to that of complex PTSD cases as opposed to more focused and specified PTSD incident(s). In the former, awareness emerges layer by layer as though peeling off the skin of the onion, ultimately freeing the patient from the emotional burden accumulated over a lengthy period of time. In the latter incident-specific cases, remediation can occur quite rapidly.

Many traumatic events (e.g., car accidents, natural disasters, etc.) are of time-limited duration. However, in some cases people experience chronic trauma that continues or repeats for months or years at a time. The current PTSD diagnosis often does not fully capture the severe psychological harm that occurs with prolonged, repeated trauma. People who experience chronic trauma often report additional symptoms alongside formal PTSD symptoms such as changes in their self-concept and the way they adapt to stressful events.

Standard evidence-based treatments for PTSD are thought to be effective for treating PTSD that occurs following chronic trauma. At the same time, treating Complex PTSD often involves addressing interpersonal difficulties. ${ }^{13}$ We view the CFS condition as similar to the Complex PTSD situation and expect progress to occur gradually rather then immediately. This is exemplified in the following case study. While Lynn's account is quite lengthy, having taken place over a four-month period of time, we believe that it is necessary to include it in its entirety to assist therapists to fully understand the treatment process. Dr. Lindenfeld's responses to the patient are italicized and indented.

My name is Lynn and I decided to re-contact Dr. George Lindenfeld after years of suffering from my Chronic Fatigue Syndrome/ Fibromyalgia condition. I have tried everything possible to address this situation including investing in my own personal flotation tank that I maintain in my garage. I had successfully consulted with Dr. Lindenfeld earlier in my life when I was dealing with some issues with my child and my emotionally abusive first husband. In desperation, I thought he might be able to direct me to obtain some type of relief.
Talk about the universe having a plan for me, Dr. Lindenfeld was engaged in the development of a study related to Chronic Fatigue Syndrome/Fibromyalgia precisely at the time I contacted him. He advised me to meet with Dr. Katherine Billiot in Ormond Beach, Florida in order to have psychometric testing done prior to beginning my exploration of RESET Therapy.

I did this computer-based testing with Dr. Billiot and then had my first RESET Therapy session with her. At first, I thought it didn't work because pain still coursed throughout my body. But then I realized that during the RESET session I was focusing specifically on my left shoulder blade where my pain was the worst. Amazingly, it dawned on me that the particular spot I had 'tuned into' didn't hurt anymore.

The weekend following the treatment, we went to an RV show and spent two hours climbing about and exploring the vehicles with no Fibro flare!!!! A big change for me. I have been so disappointed and disheartened previously when we explored RV's because I felt we could never have the travel trailer lifestyle we want when we retire. I now have hope for that dream again!

Lynn, remember back when things were 'normal'. Another way to look at it is 'pain free' time. Perhaps it begins in the number of hours in a day. Then in days per week. Perhaps you can start recording this on a calendar or in a diary to look for trends. Also, as 'good time' happens, become aware of the amount of time you can devote to chores before your inner mind starts complaining. I reference your subconscious mind as your 'automatic pilot'. I would like you to connect with that part of you so that it can come to guide you through this restoration process. P.S, remember my words of caution about over doing it!

I decided to order a BAUD to use at home, planning to focus on my whole body when our machine comes! I did have some emotional stuff come up. I felt very angry at my pain and stuff about my Mom bubbled up. She had Fibromyalgia and I believe she passed from complications from taking Lyrica.

I felt very betrayed by my body and sad that I can't do so many things that I want to do anymore. I felt quite emotional for maybe 10 minutes and then it passed. I wasn't expecting that. I'm sure it's because I try to never complain about my pain and I squash that hard! Who wants to hear someone complain constantly about the same old thing? I am hopeful that I can do more to get rid of my pain. The BAUD is due to arrive tomorrow.

We received the BAUD on Tuesday night and following Dr. Lindenfeld's RESET Therapy protocol, I set it for pain. This time I did not feel emotional as I had in Dr. Billiot's office. I concentrated on the pain in my shoulders and I got about $80 \%$ relief. Very nice for me!

I understand the concept of not doing anything strenuous after reprogramming and it makes sense. I was told today by my 93 -yearold client that I seemed to have 'more of a spring in my step' today. I have had two RESET treatments since Dr. Billiot saw me a week ago. I am about to do a session now and we will see what comes up. I find myself thinking about my mom and the resentment I felt towards what opportunities my pain has robbed me of. This whole journey is super interesting; I see why you are so immersed in it!

Lynn, let's clarify - if you chase each pain location, there will be no end to it. Alternatively, if the core is related to the underlying suppressed emotions \& we clear them at the deepest levels, the positive effect should extend throughout your entire system. After we finish the trauma circuit, we then go on to the: disappointment, despair, 
depression circuit. Going for the pain will give you temporary relief of course, so do so as you need it with the realization that your primary objective is to reduce the suppressed internal emotional pressure.

I called Dr. Lindenfeld for advice because I've been confused about the direction I'm going in. I was instructed to come up with some issues in the past where people have hurt me or disappointed me and concentrate on those issues, NOT my physical pain. I concentrated on my son not speaking to me and cutting all ties for three years after he graduated High School and decided to live with his dad. Although he and I now have a wonderful relationship, and forgiveness has been extended to him now, Dr. Lindenfeld felt I could have old feelings still in there.

BINGO! Amazing! I felt deep emotions come to me in waves and just let myself let the tears roll. Periods of emotion got shorter and periods of calm got longer until it was gone. That was very intense in a good way because at the end I felt peaceful.

Pain relief occurred that night and the next two days with about an $80 \%$ reduction all over my body!!! Mental relief was felt as well. During the session I also had anger and hurt bubble up within me. Thoughts came to me such as: "what kind of parent encourages an only child to hate his Mom. What kind of person does that?" Anger and hurt towards my ex for sure that also made me cry.

I called Dr. Lindenfeld to let him know of my success! I said I felt that I was done with the son issue. He told me to explore the feelings towards my ex-husband next. I decided to tackle the hurt/anger towards ex-husband over the long term problem of trying to turn my son away from me from age two on. Wow! I expected more tears and grief feelings but felt frightened when I concentrated on my ex.

Waves of fear lasted longer than periods of calm. When twenty minutes was up I stopped but an undercurrent of fear lingered. I am never afraid to be alone but I found myself checking the lock on the front door, and even felt compelled to lock my bedroom door for the night. It passed by morning.

I realized afterward that this person had threatened me physically and abused me mentally every chance he got for MANY years. I used to be afraid in my own home back then. I did not expect fear to come up, complete surprise, thought I would feel hurt or wronged but not frightened.

I have been feeling better lately as far as pain but this did not make it better or worse and the experience with my ex-husband felt unresolved. I reported my unexpected results to Dr. Lindenfeld and he asked "what do you think I will tell you to do next?" I said "redo the frightened ex-husband session until it is resolved.' He said that I'm catching on.'

Lynn, clearly you are making progress. As you are learning, your selecting the 'target' and getting the sound to resonate with it is the key to resolution for you. My belief is that chronic stress pushed your system into an ongoing state of fight, flight or freeze. Your system has learned that it can finally rid itself of the pent-up poison. Look forward to your next discoveries that your mind guides you to and willingly shares with you.

I did another RESET Therapy session regarding my ex-husband. I got anger this time when I was expecting a re-visiting of fear. I didn't feel the fear this time. No grief this time either that I had expected. In two sessions focused on him, I have gone through fear and anger and feel cleared of both. I have had my best pain relief to date after the last session when I had the fear reaction (not counting the flu I endured between that last session and this!).
Talked with Dr. Lindenfeld about tackling my unfelt grief over the loss of my mother eight years ago. She was my best friend and my solid rock. I knew it would be a big issue to take on. He asked if I thought I was ready and I said yes and decided to wait for a day when no kids were home but my husband would be there with me for support.

I did a grief session focusing on my Mom who passed eight years ago. I don't know how long I spent but I just let it roll until I was done. It came in waves and I let it take me over and then it would pass and then come again. Funny how it reminded me of the waves of pain I experienced in childbirth: they are overwhelming in intensity, then they subside and come again. When it is done in the end I experienced calmness.

Thankfully, I finally obtained a state of peace! I dreaded doing this knowing that this would be my most intense session. When it was at its height, I just clutched my knees to my chest and rocked and sobbed. Many innocent Kleenexes were lost in this process. My husband said it was painful for him to watch.

For me it was freeing. Now when I am reminded of her, or find pictures as we pack to move, I sit and let myself shed a few tears and allow myself to miss her. I think my psyche was protecting me before as the loss was so enormous and I always wondered when I would finally really feel it.

Now I actually am having the little moments that kind of jump up and surprise me when I am going through photos and my heart aches and I miss her. It's the ok kind of normal grief I thought people have but that I seemingly stuffed along with other 'stuff.' I suspect these feelings are the kind that probably always accompanies a great loss and I am relieved in a way to have them. It was quite odd to have felt nothing about it for so long.

It is now three weeks since I did my grief session. My fibromyalgia muscle pain has been markedly reduced to almost none. I still get pain from my back and neck needing chiropractic adjustment but the muscle pain is perhaps a mild 1 or 2 at the worst on a scale of 1-10 and often 0 . I have only used my float tank every other Saturday when my husband is working. Previous to the RESET sessions I was in every 2-3days just to stay at a manageable level of pain. I do not feel I have chronic pain in my muscles now, just occasional pain if I overdo.

We are getting ready to move and I have been packing and currently my back and neck are about a 9 level until I can get to the chiropractor on Monday. This pain is different from the Fibro pain. I can tell because if the float tank eases the pain then it is muscular in nature and I can feel the difference at the end of the float as only my spine pain stays and muscle pain is gone. I floated last night as my neck is especially bad and got no pain relief from it which tells me the pain I have today is spinal in nature, not muscular.

My take on this process is that I am a 'stuffer' of my pain and it has manifested itself this time (previously I suffered from panic attacks from stuffing my pain) as chronic muscle pain. I think all my pain bubbled up and manifested itself as tension in my muscles. That is why my frequent use of the float tank could temporarily ease that muscle pain with the warm water, weightlessness, and Epsom salts letting those muscles relax.

Without ridding myself of the internal pain however, the external pain always returned. I have been amazed at how my expectations in preparing for a session were often supplemented by feelings and situations I had not even consciously thought of for a very long time. The really buried stuff was the most interesting and obviously the stuff I needed to release the most. 
Dr. Lindenfeld asked me to summarize my RESET Therapy experience at the conclusion of my treatment process. To put it in a few words, I'm feeling immense relief from my physical pain and calmness internally. The arthritis in my back and neck continue to require chiropractic care but my muscle pain is almost completely gone. I find it still manifests in morning muscle stiffness, or stiffness when I sit too long in one spot. Amazingly, it is now quite different from the constant and chronic muscle pain I experienced before I began treatment. The fact that my float tank use has decreased from every 2-3days to none in the last month is the biggest change factor that I can reportcoming out of this experience.

I note that I am more at peace in my thoughts and have been able to take a very different tact in dealing with frustrations and disappointments that occur in my life. I am now able to step back and more realistically see that I cannot change others and it is not serving either my psyche or my tense muscles to try to continue to do so. RESET Therapy has given me the gift of letting go of these emotional triggers that caused me so much physical, and as I've learned, emotional pain.

I have experienced first-hand just how linked my feelings and my body are. I believe that this treatment has given me a way to release my stuck emotions (which is a gift in itself) and by doing so I have been able to release the chronic physical pain in my muscles. Will I never suffer another bad day of muscular pain? I have and I will probably still encounter bad days. The difference is that now I have a way to deal with them. As I get better at personally understanding exactly how my mind can control reactions in my body (both positively and negatively) I believe that I will continue to improve. RESET Therapy has simply been an amazing intervention that permits me to sustain wellness in my life.

Lynn was provided with a pre $(3 / 05 / 16)$ and post-treatment $(6 / 25 / 16)$ computer scored Pain Coping Inventory (PCI) and the Revised Fibromyalgia Impact Questionnaire (FIQR). ${ }^{14}$ The Pain Coping Inventory (PCI) is a 92-item self-report test that assesses the behavioral, cognitive, and psychological dimensions of chronic pain and takes some 20-30minutes to complete. The PCI provides 18 scales that are grouped into five major dimensions including: Physical and Temporal Qualities, Behavioral Interference, Psychological Maladjustment, Psychological Coping and Health Related Behavior. ${ }^{15,16}$

Additionally, this computer-scored inventory provides data about a patient's tendency to exaggerate or magnify pain symptomatology. This is accomplished through the review of global indices of impairment and pain generalization, nociception, coping success and treatment resistance and alienation. On this assessment tool, t-scores are used for interpretation. A normative t-score is 50 with a standard deviation of 10 . Because the normative group consisted of chronic pain patients, $t$-scores above 60 are considered to be elevated.

Factor analysis of Lynn's PCI resulted in five factor scores. The patient's Alienation, Depression and Hostility pre-treatment t-score of 42.1 and post-treatment score of 22.5 indicated that depressive symptoms and hostility were significantly reduced and minimally contributing to her perception of pain. The PCI Physical Severity and Interference score measures the severity of the patient's pain. It also summarizes the extent to which muscle bracing/movement phobia, sleep disturbance, and interference of activities/avoidance contributes to her symptoms. The results from her pre-treatment administration of the Physical Severity and Interference score was 44.5 , with a posttreatment score of 33.3 .
The PCI General Psychological Adjustment score summarizes the patient's positive coping mechanisms and indicates how well the patient is adjusting to her chronic pain condition. On this score, the higher the result, the better the coping ability. Lynn received a pre-treatment t-score of 44.5 on this factor with a post-treatment score of 80.6 indicating that she had adjusted exceedingly well to her condition. The final PCI factor score, Psychological Orientation and Motivation, generally assesses the degree to which a patient will respond to psychological interventions and to her motivation for treatment. She received a pre-treatment t-score of 48.7 with a posttreatment score of 96.7 on this factor indicating that she was now an excellent candidate for psychological intervention.

In regard to the validity of the test results, Lynn's PCI Extreme Belief Frequency pre-treatment score was 42.1 with a posttreatment score of 12.7 , revealing a highly diminished tendency to view pain related symptoms at an extreme level. The t-score results on the EBF indicates the extent to which the complaint of pain is coming primarily from documented organic/physical pathology as opposed to psychological factors such as depression, anger, anxiety, hypochondriasis, conversion hysteria, character pathology, or secondary gain motivations.

The obtained results suggested that the symptom magnification which previously impacted her perception now colored her responses minimally and was no longer contributing to her disablement. Finally, her PCI Global Impairment Index pre-treatment score of 42.1 as compared to her Post-treatment score of 23.7 revealed an overall significant improvement in her condition. Because of the extensive changes seen in this patient as a result of her RESET Therapy treatment involvement, the 18 primary scales are provided here for comparative purposes.

\section{Physical \& temporal severity scales}

As evidenced in the above three scales, dramatic changes were apparent in all three domains. Pain Lability was reduced to $0 \%$ with Pain Severity dropping by $76 \%$ and Pain Continuity \& Duration reduced by $50 \%$.

\section{Behavioral interference scales}

On the Behavioral Interference Scales, one of the most dramatic finding in the test battery was found on the Sleep Disturbance Scale with $3 / 05 / 16$ results at $50 \%$ reducing to $4.2 \%$ on $6 / 25 / 16$. This was followed by a $60 \%$ reduction on the Muscle Bracing, Movement Phobia Scale.

\section{Psychological malajustment scales}

On the Psychological Maladjustment Scales, Stress and Anxiety elevated from 32.5 to 37.5 . Significant drops are evident on the Depression, Hostility and Catastrophizing Scales. The Paranoia \& Alienation Scale dropped by around 25\%.

\section{Psychological coping scales}

On the Psychological Coping Scales, two scales demonstrated highly significant changes. The first (Perceived Self-Efficacy), increased from 26.2 to 93.8. The second (Psychological Motivation), changed from 25.1 to 97.2. Her Quality of Life score rose from 41.1 to 80 .

\section{Health related behavior scales}

Positive trends are also evident on the Health Related Behavior Scales with a dramatic change noted on the Chemical Independence scale. 
The Fibromyalgia Impact Questionnaire (FIQ) was used initially in the evaluation of fibromyalgia (FM) patients. ${ }^{17}$ The FIQR was developed in response to known deficiencies of the FIQ with the help of a patient focus group. ${ }^{14,18}$ The FIQR has the same 3 domains as the FIQ (that is, function, overall impact and symptoms). It differs from the FIQ in having modified function questions and the inclusion of questions on memory, tenderness, balance and environmental sensitivity. All questions are graded on a $0-10$ numeric scale.

The scoring of the FIQR includes: the summed score for Function (range 0 to 90 ) divided by 3; the summed score for Overall Impact (range 0 to 20) is unchanged; the summed score for Symptoms (range 0 to 100 ) is divided by 2 . The total FIQR is the sum of the three modified domain scores. The weighting of the total score includes $30 \%$ as ascribed to 'Function'; $50 \%$ is ascribed to 'Symptoms'; 'Overall Impact' remains at $20 \%$. The total maximal score of the FIQR remains at 100 .

The total average score of the FIQR in Fibro Myalgia patients was $56.58 \pm 20$ (range 15 to 97 ) with a median score of 58 . As noted in Lynn's pre-treatment scores, her total score of 57.5 was in line with the average scoring level for others with her condition. Her Functioning score was on the lower end of the continuum on this index. Alternatively, her score of 18 on the Overall Impact score was at the upper level on this particular index score. Her Symptom score of 25.5 was midway on this index.

Post-treatment scores over a four-month course of RESET Therapy revealed dramatic changes in her condition, the most impressive of which was her Overall Impact index score of 1, indicating an astonishing reduction of $94 \%$ in this category. Her Functioning index score was reduced by $50 \%$ and her Symptom index score reduced by an amazing $80 \%$.

\section{Revised fibromyalgia impact questionnaire (FIQR)}

A compelling flow of neuroscientific research is now providing support for our hypotheses. We detail a sampling of findings from varied research domains that seek to address the Chronic Fatigue Syndrome issue. This review is not intended to be comprehensive or complete. Rather, we seek to touch on the many facets of current inquiry and to speculate as to whether they have been productive or not.

Reference to a number of seminal articles are available on the healthrising.org website pertaining to varied CFS issues such as Cognition and Chronic Fatigue Syndrome. ${ }^{19}$ Regarding cognitive impairment, our cumulative clinical experience suggests that many persons with CFS have difficulty with memory, verbal articulation as well as verbal comprehension, multitasking and information processing speed. As you would guess, similar data is associated with the PTSD condition. ${ }^{20}$

A small study ${ }^{6,7}$ reported direct evidence of inflammation in the brain of Myalgic Encephalomyelitis (ME)/Chronic Fatigue Syndrome (CFS) patients. Previously, critics of ME/CFS referred to a lack of evidence for the referred inflammation aspect of the condition. The investigators used PET and MRI imaging to observe brain processing through the use of radioactive tracers that bind to activated microglia (the main immune cells of the brain).

The results showed that neuroinflammation markers were higher for patients than controls across many brain areas including the thalamus, the pons and the midbrain including the amygdala. They also found that the severity of symptoms correlated with the degree of inflammation in multiple brain regions, particularly for cognitive functioning. It was the correlation between a biological findingneuroinflammation-and clinical problems that Dr. Anthony Komaroff of the Massachusetts CFIDS/ME \& FM Association found so exciting about this work, because it suggested a biologically plausible explanation for the symptoms of ME/CFS: ${ }^{21}$

If replicated it would, for me, say that there is a low-grade, chronic encephalitis in these patients, that the image we clinicians have of encephalitis as an acute and often dramatic clinical presentation that can even be fatal has - may have - blinded us to the possibility that there may be that long-lasting - many years long — cyclic chronic neuroinflammation that is underlying the symptoms of this illness.

We believe that the importance of the above research cannot be understated: it dispels the notion that CFS is a feigned illness utilized by malingers or those with primary mental illness. Our first paragraph, above, observed that: "No one knows what causes CFS." Perhaps further research such as that produced by Nakatomi et al., can finally provide substance to replace erroneous beliefs about this debilitating condition.

A number of factors have been investigated as possible causes of CFS. The first focuses on disease because some people have developed CFS after having a viral infection. ${ }^{22}$ Among those viruses considered as possible triggers are Epstein-Barr virus, ${ }^{23}$ human herpes virus $6,{ }^{24}$ and mouse leukemia viruses. ${ }^{25}$ No conclusive link has been found and rather, this speculation in regards to viral infection and CFS has been debunked. ${ }^{26}$

Seemingly, the above avenue of focus has dead ended with no productive data emanating from rather extensive and ongoing research efforts. While the effort will likely continue due to the 'if it doesn't work try harder' effect, for our purposes, we perceive that the quest for disease causation of CFS is no longer a viable avenue of exploration.

We next explore the area of immune system problems. ${ }^{27,28}$ Some individuals with weakened immune systems also evidence CFS. However, it is unclear whether this aspect is causative or simply accompanies the primary condition. Also hormonal blood levels have been found to be abnormal in the hypothalamus, pituitary glands or adrenal glands. The significance of these findings is unknown, ${ }^{29}$

The chicken or the egg question becomes pertinent here. We are inclined to speculate that CFS emerges consequent to prolonged stress due to the absence of positive mechanisms through which the patient might release pent-up emotionally charged material. Our perspective is that chronically containing this material further weakens an already compromised immune system.

We now shift focus to brain structure differences found between those with CFS compared to a normative population of subjects. Since the early 1990s, fMRI has come to dominate brain mapping research because it does not require people to undergo shots, surgery, or to ingest substances, or be exposed to ionising radiation, etc. ${ }^{30}$ An example of CFS research that is associated with differences in brain structure in CFS patients as compared to non CFS patients is found in conclusions drawn by Dr. Michael M. Zeineh of Stanford University. ${ }^{31,32}$ Zeineh noted that:

It's not uncommon for CFS patients to face several mischaracterizations of their condition, or even suspicions of hypochondria, before receiving a diagnosis of CFS. Findings of the study reveal that: The brains of patients with chronic fatigue syndrome have diminished white matter and white matter abnormalities in the right hemisphere. . . Using a trio of sophisticated imaging methodologies, we found that CFS patients' brains diverge from those of healthy subjects. 
The topic of brain dysregulation as evidenced in QEEG and Loreta is an emerging area of interest. To begin with, electroencephalography (EEG) is the measurement of electrical patterns at the surface of the scalp which reflect cortical activity, which is commonly referred to as 'brainwaves'("QEEG Assessment Quantitative Electroencephalography - Listen And Learn Centre," n. d.) Quantitative EEG (qEEG) is the analysis of the digitized EEG, and in lay terms, this sometimes is also called 'Brain Mapping'. The qEEG is an extension of the analysis of the visual EEG interpretation which may assist and even augment our understanding of the EEG and brain function.

New advances in technology are giving investigators a deeper look at brain inflammation early in the course of the CFS condition. ${ }^{27}$ The Nakatomi study was among the earliest, ${ }^{6,7}$ found evidence of neuroinflammation in the cingulate cortex, hippocampus, amygdala, thalamus, midbrain, and pons regions of the brain.

The fact that the degree of the widespread neuroinflammation was strongly correlated with the severity of the fatigue, pain, cognitive problems and depression found in the ME/CFS group was encouraging. The areas seemed to fit what we know about ME/CFS. One inflamed area regulates the reticular activating system which determines how awake or aroused one is. Several others are involved in pain sensitivity, cognitive impairment and mood.

Research inquiries such as those described above that utilize fMRI, QEEG, Loreta, etc., are opening windows where before there were walls. Theories that endure the test of time are built on solid repeatable, factually based information. Our RESET Therapy underpinnings have been developed, refined and now applied to varied conditions that extend beyond the effects of emotional trauma. We look now at exciting research about genetic changes in receptors that is bearing fruit. The results of inquiries such as this help us to understand how those who have experienced chronic trauma manifest an anxiety signature into future generations..$^{33,34}$

Co-researcher and consultant immunologist Professor Pete Smith said that important signaling mechanisms are disrupted as a result of these genetic changes involving the detection and response to threats. These are primitive genes that are involved in many cellular signals in the brain, gut, cardiovascular and immune systems, as well as in the mediation of pain

Our final area of inquiry is a study published in Molecular Psychiatry byMady Hornig, MD, and colleagues used immunoassay testing methods. ${ }^{33,35}$ The investigators found that an inflammatory immune molecule. . . was depressed in individuals with ME/CFS compared with the other two groups, matching what was seen in the blood study in patients who had the disease for more than three years.

We now know that the same changes to the immune system that we recently reported in the blood of people with ME/CFS with long-standing disease are also present in the central nervous system. . . These immune findings may contribute to symptoms in both the peripheral parts of the body and the brain, from muscle weakness to brain fog.

\section{Summary}

A monumental tipping point was noted in the Annals of Internal Medicine 2015 position wherein three national panels (IOM, AHRQ, and $\mathrm{NIH}$ ) accepted evidence of various neurologic abnormalities in patients with ME/CFS ${ }^{2}$. No longer do those with this diagnosis, or others like it, have to live in a world of 'shadow syndromes.' The CDC stated that "There is no cure for CFS, so the goal of treatment is to improve symptoms". ${ }^{1}$ We believe that this statement may prove to be erroneous and seek to find therapeutic interventions that establish remediation for those impaired with CFS.

We believe that there is a built in genetic redundancy in our cellular material with evidence for this perspective emerging from studies of the cycle of the cell, immunology and neurobiology. ${ }^{26}$ The point to be made here is that, if a particular intervention is able to remediate brain inflammation for a specified condition such as PTSD, then it would seem reasonable to inquire if the same intervention would produce similar results in other such conditions within the same genetically structured organism.

This paper began with a series of questions regarding the possibility of linkage between two vastly different diagnoses that evidenced some similarities in their respective manifestations, both of which include the presence of brain inflammation. We further proposed that two critical components of RESET Therapy are common to all emerging memory and trauma based therapies. Re-stimulating the reactive limbic portion of a selected neural network, thereby 'lighting up' the sustained traumatic material or emotionally charged, was one of the primary factors. The second aspect involved the therapist's introduction of a modifying stimulus that would alter the involved neuronal network. ${ }^{36,37}$

A detailed case study was provided with a patient's self-report describing her self-use of RESET Therapy to remediate fatigue and accompanying pain symptomology. Lynn's treatment pattern was similar to that of other CFS patient's in that it required a significant period of time (4-months) to alter her rigidified pattern. As noted earlier, the treatment format was closer to the Complex PTSD model than it was to the incident specific one. Part of the adjustment process involved shifting focus from specific pain location to inwardly contained, emotionally suppressed material. As seen in her progress notes, increasing levels of insight emerged as the contained and suppressed material drained from her system. Also note, to her surprise, the emergence of emotional material that she believed was long resolved but remained suppressed and in fact, was unresolved.

Lynn's positive results from her course of treatment lends tentative support to one of our main hypothesis. Specifically, we perceived that with the introduction of a stimulus that interfered with the memory reconsolidation process, a neuronal system reaction would take place that would transform the afflicted individual from a chronic defensive/ reactive mode to that of a normative state of growth. Thus our earlier questioning pertaining to a possible correspondence between CFS and PTSD appears to have some provisional support. Supportive withinpatient reference as measured through psychometric means include significant changes in her pre and post-test scores. The exception in her scoring pattern was noted on the Stress \& Anxiety scale of the PCI. We perceive that this scale is ratedhigher at the completion of the patient's course of treatment because she is now more aware of previously suppressed emotional material and is reacting accordingly.

If these conclusions receive further support through additional studies that might use fMRI, qEEG, etc., we will be able to affirm that the symptoms associated with Chronic Fatigue Syndrome also interweave closely with memory circuits in the limbic system of the brain. We will need time to ascertain if Lynn's neural reset is sustained. If this does occur, RESET Therapy can be explored further to offer remediative hope to those who continue to suffer from the deleterious effects of CFS.

In addition, other ailments that reference inflammatory aspects may also be amenable to remediation through a RESET Therapy 
approach. In line with this thinking, our next exploratory focus will be that of the Irritable Bowel Syndrome condition. If our initial CFS case study findings are similar to our future IBS case study findings, a unified theory pertaining to our focus on neuronal networks as a primary means of addressing previous ailments of unknown origin will begin to shed light on a possible causation factor in these and other conditions that were previously thought of as being untreatable.

\section{Acknowledgments}

None.

\section{Conflicts of interest}

Author declares there are no conflicts of interest.

\section{Funding}

None.

\section{References}

1. Centers for Disease Control and Prevention. Chronic Fatigue Syndrome. 2014

2. Komaroff AL. Myalgic Encephalomyelitis/Chronic Fatigue Syndrome: A Real Illness. Annals of Internal Medicine. 2015;162(12):871-872.

3. Charles W. CFS versus FM: Twins, Cousins, or Just Acquaintances? CFIDS \& Fibromyalgia Self-Help. 2017.

4. Romano GF, Tomassi S, Russell A, et al. Fibromyalgia and Chronic Fatigue: The Underlying Biology and Related Theoretical Issues. $A d v$ Psychosom Med. 2015;34:61-77.

5. Julius HB, Richard ML, Peter DW. The common link between functional somatic syndromes may be central sensitisation. 2015;78(3):228-236.

6. Logan R. Nakatomi: neurological inflammation in ME/CFS. 2015.

7. Nakatomi Y, Mizuno K, Ishii A, et al. Neuroinflammation in Patients with Chronic Fatigue Syndrome/Myalgic Encephalomyelitis: An 11C-(R)-PK11195 PET Study. J Nucl Med . 2014;55(6): 945-950.

8. Lindenfeld G, Bruursema LR. Resetting the Fear Switch in PTSD: A Novel Treatment Using Acoustical Neuromodulation to Modify Memory Reconsolidation. 2015.

9. Lindenfeld G, Rozelle G. PTSD: Brain on Fire: A RESET Therapy (QEEG) Brain Map Analysis of an Afghanistan Combat Veteran. Academia.edu. 2015.

10. Kumar S, von Kriegstein K, Friston K, et al. Features versus feelings: dissociable representations of the acoustic features and valence of aversive sounds. The Journal of Neuroscience: The Official Journal of the Society for Neuroscience. 2012;32(41):14184-14192.

11. Narayanan RT, Seidenbecher T, Kluge C, , et al. Dissociated theta phase synchronization in amygdalo- hippocampal circuits during various stages of fear memory. Eur J Neurosci. 2007;25(6):1823-1831.

12. Perusini JN, Meyer EM, Long VA, et al. Induction and Expression of Fear Sensitization Caused by Acute Traumatic Stress. Neuropsychopharmacology. 2016;41(1):45-57.

13. Complex PTSD. Complex PTSD. PTSD: National Center for PTSD. 2016.

14. Bennett RM, Friend R, Jones KD, et al. The Revised Fibromyalgia Impact Questionnaire (FIQR): validation and psychometric properties. Arthritis Res Ther. 2009;11(5):415.
15. Hadjistavropoulos HD, MacLeod FK, Asmundson GJ. Validation of the Chronic Pain Coping Inventory. Pain. 1999;80(3):471-481.

16. Pain Coping Inventory (PCI).

17. Bennett R. The Fibromyalgia Impact Questionnaire (FIQ): a review of its development, current version, operating characteristics and uses. Clin Exp Rheumatol. 2005;23(5 Suppl39):S154-S162.

18. Bennett RM, Friend R, Jones KD, et al. The Revised Fibromyalgia Impact Questionnaire (FIQR): validation and psychometric properties. Arthritis Res Ther. 2009;11(4):R120.

19. Johnson C. Cognition and Chronic Fatigue Syndrome (ME/CFS): Key Findings. 2016.

20. Wrocklage KM, Schweinsburg BC, Krystal JH, et al. Neuropsychological Functioning in Veterans with Posttraumatic Stress Disorder: Associations with Performance Validity, Comorbidities, and Functional Outcomes. $J$ Int Neuropsychol Soc. 2016;22(4):399-411.

21. Komaroff AL. Dr. Komaroff's Progress Report on Chronic Fatigue Syndrome Research. 2008.

22. Addressing Hidden Viral Infections in CFS/FMS Can Sometimes Be Helpful. Vitality. 2007;101.

23. Phoenix Rising. Epstein Barr Virus I: EBV Rides Again. 2012?

24. Eymard D, Lebel F, Miller M, et al. Human herpesvirus 6 and chronic fatigue syndrome. Can J Infect Dis. 1993;4(4):199-202.

25. Lo SC, Pripuzova N, Li B, et al. Detection of MLV-related virus gene sequences in blood of patients with chronic fatigue syndrome and healthy blood donors. Proc Natl Acad Sci U S A. 2010;107(36):15874-15879.

26. Nowak MA, Boerlijst MC, Cooke J, et al. Evolution of genetic redundancy. Nature. 1997;388(6638):167-171.

27. Hornig M, Montoya JG, Klimas NG, et al. Distinct plasma immune signatures in ME/CFS are present early in the course of illness. Science Advances. 2015;1(1):e1400121.

28. Thompson D. Immune System Changes Tied to Chronic Fatigue Syndrome. 2015.

29. Cleare AJ, Bearn J, Allain T, et al. Contrasting neuroendocrine responses in depression and chronic fatigue syndrome. J Affect Disord. 1995;34(4):283-289.

30. Andersen J. What Is Functional Magnetic Resonance Imaging. 2012?

31. Goldman B. Study finds brain abnormalities in chronic fatigue patients. 2014.

32. Zeineh MM, Kang J, Atlas SW, et al. Right arcuate fasciculus abnormality in chronic fatigue syndrome. Radiology. 2015;274(2):517-526.

33. Aussie scientists find genetic changes associated with chronic fatigue syndrome (CFS). 2015.

34. Marshall-Gradisnik S, Huth T, Chacko A, et al. Natural killer cells and single nucleotide polymorphisms of specific ion channels and receptor genes in myalgic encephalomyelitis/chronic fatigue syndrome. Appl Clin Genet. 2016;9:39-47.

35. Hornig M, Gottschalk G, Peterson DL, et al. Cytokine network analysis of cerebrospinal fluid in myalgic encephalomyelitis/chronic fatigue syndrome. Molecular Psychiatry. 2016;21(2):261-269.

36. Teitelbaum J. From Fatigued to Fantastic (3rd edn). New York: Avery, USA. 2007.

37. QEEG Assessment Quantitative Electroencephalography. Listen and Learn Centre. 2014. 